\title{
INVERTIBLE MEASURE PRESERVING TRANSFORMATIONS AND POINTWISE CONVERGENCE
}

\author{
J.-M. BELLEY ${ }^{1}$
}

\begin{abstract}
An investigation of pointwise convergence of sequences $\left\{\sum_{j=-\infty}^{\infty} a_{j}^{k} f\left(T^{-j} x\right): k=1,2, \cdots\right\}$ where $f$ lies in the space $L^{1}([0,1])$ of Lebesgue integrable functions on the unit interval, $T$ is an invertible measure preserving transformation on $[0,1]$, and the sequence of polynomials $\left\{\sum_{j=-\infty}^{\infty} a_{j}^{k} z^{-j}: k=1,2, \cdots\right\}$ is uniformly bounded and pointwise convergent for all $z$ such that $|z|=1$.
\end{abstract}

Spectral properties. An invertible measure preserving transformation $T$ on the unit interval $I$ is known to induce a unitary operator on the space $L^{2}(I)$ of square integrable functions on $I[6$, p. 13]. By the spectral theorem [5, p. 71] there exists a spectral measure $E$ on the Borel subsets of the unit circle $C$ in the complex plane such that for any integer $k$, $U^{k}=\int z^{k} E(d z)$ in the sense of strong convergence. Let the resolution of the identity $E_{t}, t$ in $[0,2 \pi)$, be given by $E(\{\exp (i s): 0 \leqq s<t\})$. Then $[3$, p. 482]

$$
E_{t}=\sum_{j \neq 0} \frac{\exp (i j t)-1}{2 \pi i j} U^{-j}+\frac{t}{2 \pi}+\frac{E(\{1\})-E(\{\exp (i t)\})}{2}
$$

where, for each $z$ in $C, E(\{z\})=\lim \left(\sum_{j=-n}^{n} z^{j} U^{-j}\right) /(2 n+1)$ and the symbol $\sum_{j \neq 0}$ denotes the limit as $n$ tends to infinity of the sum $\sum_{j=-n ; j \neq 0}^{n}$.

Substituting the Fourier series

$$
\begin{aligned}
\pi-\sum_{j \neq 0} \frac{\exp (i j s)}{i j} & =s, & & 0<s<2 \pi, \\
& =\pi, & & s=0,
\end{aligned}
$$

on the right-hand side of the identity [1, p. 100]

$$
s=\pi+32 \sum_{j=0}^{\infty} \frac{\sin \left(\frac{1}{4}(2 j+1) s\right)-(-1)^{j} \cos \left(\frac{1}{4}(2 j+1) s\right)}{\pi^{2}(2 j+1)^{3}} \quad(0 \leqq s \leqq 2 \pi),
$$

and then integrating both sides with respect to the spectral measure

Received by the editors June 7, 1972 and, in revised form, June 25, 1973.

AMS (MOS) subject classifications (1970). Primary 28A65; Secondary 47A35.

${ }^{1}$ This research was supported by a grant from the National Research Council of Canada. 
for the unitary operator $\exp (-i t) U$ yields

$$
\begin{aligned}
E_{t}= & \frac{t}{2 \pi}+\frac{1}{2} E(\{1\})-\sum_{j \neq 0} \frac{U^{-j}}{2 \pi i j} \\
& -\frac{16}{\pi^{3}} \sum_{j=0}^{\infty} i \frac{\exp (-i(2 j+1) t / 4) U^{(2 j+1) / 4}-\exp (i(2 j+1) t / 4) U^{-(2 j+1) / 4}}{(2 j+1)^{3}} \\
& -\frac{16}{\pi^{3}} \sum_{j=0}^{\infty}(-1)^{j}\left\{\left(\exp (-i(2 j+1) t / 4) U^{(2 j+1) / 4}\right) /(2 j+1)^{3}\right. \\
& \left.+\left(\exp (i(2 j+1) t / 4) U^{-(2 j+1) / 4}\right) /(2 j+1)^{3}\right\} .
\end{aligned}
$$

By the uniform boundedness of the series [7, p. 18] we can justify taking the integral inside the summation signs above.

The unitary operators $U^{k / 4}, k=0, \pm 1, \pm 2, \cdots$, are defined by $U^{k / 4}=\int z^{k / 4} E(d z)$. Thus the convolution property for the spectral measure of a unitary operator with the multiplicative property $[4, \mathrm{pp} .639,640]$ permits us to establish that, since $U$ is multiplicative, then so is $U^{k / 4}$. For if $f, g$ and their product $f g$ lie in $L^{2}(I)$ then

$$
\begin{aligned}
U^{k / 4} f g & =\int z^{k / 4} E(d z) f g=\iint z^{k / 4} E\left(w^{-1} d z\right) f E(d w) g \\
& =\int\left(\int\left(w^{-1} z\right)^{k / 4} E\left(w^{-1} d z\right) f\right) w^{k / 4} E(d w) g=\left(U^{k / 4} f\right)\left(U^{k / 4} g\right) .
\end{aligned}
$$

Hence if $f$ lies in $L^{2}(I)$ with $L^{1}$ norm $\|f\|_{1}$ then there exists $g$ in $L^{2}(I)$ with $L^{2}$ norm $\|g\|_{2}$ such that $f=g^{2},\|f\|_{1}=\|g\|_{2}^{2}$, and $\left\|U^{k / 4} f\right\|_{1}=\left\|U^{k / 4} g\right\|_{2}^{2}=$ $\|f\|_{1}$. Using the identity above for $E_{t}$ it now follows that there exists a constant $K$ such that for any collection $\left\{B_{m}: m=1,2, \cdots\right\}$ of disjoint half-open interval subsets of $C$ and any $f$ in $L^{2}(I)$ we have $\left\|E\left(\bigcup B_{m}\right) f\right\|_{1} \leqq$ $K\|f\|_{1}$. By the usual measure theoretic argument (Dinculeanu [2]), for any $f$ in $L^{2}(I)$ and any Borel subset $B$ of $C,\|E(B) f\|_{1} \leqq K\|f\|_{1}$. Since $L^{2}(I)$ is dense in $L^{1}(I)$ we extend by continuity the operator $E$ to $L^{1}(I)$ and so (retaining the symbol $E$ for the extension) $\|E(B) f\|_{1} \leqq K\|f\|_{1}$ for all $f$ in $L^{1}(I)$ and Borel subset $B$ of $C$. Note that the space $L^{\infty}(I)$ of essentially bounded functions on $I$ lies in $L^{2}(I)$. Hence $E$ is defined on $L^{\infty}(I)$. We now deduce that for any $h$ in $L^{\infty}(I)$ with $L^{\infty}$ norm $\|h\|_{\infty}$ and any Borel $B$ in $C$, $\|E(B) h\|_{\infty} \leqq K\|h\|_{\infty}$. For if $f$ lies in $L^{1}(I)$, using $(f, h)$ to denote the integral of the product $f h$ (where $h$ is the complex conjugate of $h$ ) over $I$, we get $(E(B) f, h)=(f, E(B) h)$ which is clear if $f$ lies in $L^{2}(I)$ and extends to $L^{1}(I)$ by continuity.

Next let us show the existence of a constant $K^{\prime}$ such that for any $h$ in $L^{\infty}(I)$ and any sequence $\left\{B_{k}\right\}$ of disjoint Borel subsets of $C$, $\left\|\sum\left|E\left(B_{k}\right) h\right|\right\|_{\infty} \leqq K^{\prime}\|h\|_{\infty}$. Otherwise there would exist some finite family 
$\left\{B_{k}: k=1,2, \cdots, n\right\}$ of disjoint Borel subsets of $C$ such that for some $h$ in $L^{2}(I), \sum\left|E\left(B_{k}\right) h\right|$ is "much" greater than $\|h\|_{\infty}$ on some subset $X$ of $I$ of positive measure. Hence by considering the real and imaginary parts of $E\left(B_{k}\right) h$ and all possible subsequences of $\left\{B_{k}: k=1,2, \cdots, n\right\}$, we see that there must exist some subsequence $\left\{B_{k_{j}}\right\}$ for which either the real or imaginary part of $E\left(\cup B_{k_{i}}\right) h$ is "much" greater in absolute value than $\|h\|_{\infty}$ on a subset of $X$ of positive measure, i.e. $\left\|E\left(\bigcup B_{k_{j}}\right) h\right\|_{\infty}>K\|h\|_{\infty}$ which is a contradiction.

By now we have that for any given $h$ in $L^{\infty}(I), E(\cdot) h(x)$ is a complex measure on the Borel subsets of $C$ with total variation not exceeding $K^{\prime}\|h\|_{\infty}[8$, p. 117] for almost all $x$ in $I$. Hence we can define in the usual way the integral $\int q(x, z) E(d z) h(x)$ of a bounded Borel measurable function $q(x, z)$ on $I \times C$ to yield an essentially bounded function of $x$, i.e. an element of $L^{\infty}(I)$. Furthermore if $\left\{q_{k}(x, z)\right\}$ is a pointwise convergent uniformly bounded sequence of Borel measurable functions then by Lebesgue's dominated convergence theorem the integrals $\int q_{k}(x, z) E(d z) h(x)$ form a uniformly bounded (in $L^{\infty}(I)$ ) almost everywhere pointwise convergent sequence of functions on $I$.

Convergence properties. Consider a sequence of polynomials $p_{k}(z)=$ $\sum_{j=-\infty}^{\infty} a_{j}^{k} z^{-j}, k=1,2, \cdots$, where $z$ lies in $C$ and $a_{j}^{k}$ are complex coefficients all but a finite number of which vanish. For a given function $f$ in $L^{1}(I)$ define $p_{k}(U) f$ to be $\sum_{j=-\infty}^{\infty} U^{-j}\left(a_{j}^{k} f\right)$, i.e. $\sum_{j=-\infty}^{\infty} a_{j}^{k} U^{-j} f$.

THEOREM. If $U$ is an operator on $L^{1}(I)$ induced by an invertible measure preserving transformation on the unit interval $I$ and $\left\{p_{k}(z): k=1,2, \cdots\right\}$ a pointwise convergent sequence of uniformly bounded (trigonometric) polynomials on the unit circle then, for all $f$ in $L^{1}(I), p_{k}(U) f(x)$ converges pointwise for almost all $x$ in $I$ as $k$ tends to infinity.

Proof. If $p_{k}(U) f$ does not converge pointwise almost everywhere, there exists a nonzero constant $d$ such that for all $x$ in a subset $Y$ of $I$ of positive measure $|Y|$

$$
\sup _{k^{\prime}, k^{\prime \prime} \geqq m}\left|p_{k^{\prime}}(U) f(x)-p_{k^{\prime \prime}}(U) f(x)\right|>d
$$

for all integers $m$. Hence given any $m$ there exists an integer $M>m$ and measurable functions $k^{\prime}(x), k^{\prime \prime}(x) ; m \leqq k^{\prime}(x), k^{\prime \prime}(x) \leqq M$ such that for some function $h,|h|=1$, we have

$$
\left(\sum_{j} U^{-j}\left(a_{j}^{k^{\prime}(x)}-a_{j}^{k^{\prime \prime}(x)}\right) f(x), h(x)\right)>\frac{d|Y|}{2} .
$$


Note that $M$ was chosen to make

$$
\sup _{m \leqq k^{\prime}, k^{\prime \prime} \leqq M}\left|p_{k^{\prime}}(U) f(x)-p_{k^{\prime \prime}}(U) f(x)\right|>d
$$

for all $x$ in a subset of $Y$ of measure greater than $|Y| / 2$. But by the measure preserving property of the operator inducing $U$ we have

$$
\begin{gathered}
\left(\sum_{j} U^{-j}\left(\left(a_{j}^{k^{\prime}(x)}-a_{j}^{k^{\prime \prime}(x)}\right) f(x)\right), h(x)\right)=\left(f(x), \sum_{j}\left(a_{j}^{k^{\prime}(x)}-a_{j}^{k^{\prime \prime}(x)}\right) U^{j} h(x)\right) \\
=\left(f(x), \int \sum\left(a_{j}^{k^{\prime}(x)}-a_{j}^{k^{\prime \prime}(x)}\right) z^{j} E(d z) h(x)\right)
\end{gathered}
$$

and by the discussion at the end of the previous section this tends to zero as $m$ tends to infinity, which is a contradiction. Q.E.D.

The above could be generalized to not necessarily invertible transformations on the real line, which would make Birkhoff's ergodic theorem [6, p. 18] a special case of the theorem above by taking the polynomial $\sum_{j=-k}^{k} z^{-j} /(2 k+1)$ for $p_{k}(z)$. In fact we could go even further by considering operators which are $L^{1}$ and $L^{2}$ contractions with the multiplicative property by using the generalized spectral measures associated with them [9, pp. 12-18].

\section{BIBLIOGRAPHY}

1. H. Davis, Fourier series and orthogonal functions, Allyn and Bacon, Boston, Mass., 1963.

2. N. Dinculeanu, Vector measures, Pergamon Press, New York, 1967.

3. J. L. Doob, Stochastic processes, Wiley, New York; Chapman \& Hall, London, 1953. MR 15, 445.

4. C. Foiaș, Sur les mesures spectral qui interviennent dans la théorie ergodique, J. Math. Mech. 13 (1964), 639-658. MR 29 \#215.

5. P. R. Halmos, Introduction to Hilbert space and the theory of spectral multiplicity, Chelsea, New York, 1951. MR 13, 563.

6. - Lectures on ergodic theory, Publ. Math. Soc. Japan, no. 3, The Mathematical Society of Japan, Tokyo, 1956. MR 20 \#3958.

7. Y. Katznelson, An introduction to harmonic analysis, Wiley, New York, 1968. MR 40 \#1734.

8. W. Rudin, Real and complex analysis, McGraw-Hill, New York, 1966. MR 35 \#1420.

9. B. Sz.-Nagy, Extensions of linear transformations in Hilbert space which extend beyond this space, Ungar, New York, 1960. MR 22 \#8338.

Département de Mathématiques, Université de Sherbrooke, Sherbrooke, QuÉBec, CANADA 